\title{
T-Lymphocyte Activation in Adult-Onset Idiopathic Hypoparathyroidism
}

Jacobo Wortsman, M.D., Peter MCConnaChIE, Ph.D., Springfield, Illinois,

JAMES R. BAKER, Jr., M.D., Ann Arbor, Michigan, LAWRENCE E. MALLETTE, M.D., Ph.D.,

Houston, Texas

PURPOSE: Patients with adult-onset idiopathic hypoparathyroidism (AOIH) often have antibodies against the parathyroid glands and other tissues, suggestive of immune activation. The purpose of this study was to determine whether T-cell activation is also a component of the endocrine disease.

PATIENTS AND METHODS: We identified eight patients with idiopathic hypoparathyroidism diagnosed after the age of 30 years at two tertiary care centers and evaluated peripheral blood lymphocyte subset phenotype frequencies using monoclonal antibodies and flow cytometry. Control subjects were 13 patients with Graves' disease (five thyrotoxic and eight euthyroid) and 110 healthy volunteers. In two of the patients with AOIH, we also determined the mitogenic response to parathyroid cell membranes in peripheral lymphocytes.

RESULTS: Patients with AOIH had higher than normal frequencies of the following phenotypes ( $p<0.05$ versus controls, one-way analysis of variance): CD4, helper T cells; CD29/CD4, inducer of helper $T$ cells; CD16 and CD56, natural killer cells; and CD3/DR, activated $T$ cells coexpressing DR. Patients with Graves' disease had significantly higher than control frequencies of CD25 ( $T$ cells bearing the interleukin-2 receptor), CD3/DR, and CD26 (also a marker of Tcell activation); whereas the frequency of CD29/CD4 was significantly less than the control frequency. Neither of the two AOIH patients tested showed lymphocyte proliferation in response to parathyroid or thyroid cell membrane fractions.

CONCLUSIONS: Generalized T-cell activation represents a novel feature associated with AOIH. Although we could not demonstrate parathyroid-specific lymphocyte clonal expansion, these data are suggestive of a generalized im-

From the Department of Internal Medicine (JW) and Medical Microbiology-Immunology (PM), Southern Ilinois University, Springfield, Ilinois; the Department of Medicine (JRB), University of Michigan, Ann Arbor, Michigan; and the Department of Medicine (LEM), Baylor College of Medicine and Veterans Administration Medical Center, Houston, Texas.

Requests for reprints should be addressed to Jacobo Wortsman, M.D., P.O. Box 19230, Springtield, Illinois 62794-9230.

Manuscript submitted August 4, 1991, and accepted in revised form November 6, 1991 . mune disturbance possibly related to autoimmunity, in which one of the manifestations is hypoparathyroidism.

$\mathbf{P}$ atients with idiopathic hypoparathyroidism have a high frequency of circulating antibodies against the parathyroid glands and other tissues [1-4]. The antibodies, detected by immunofluorescence, are mostly of the IgG and IgM isotypes, and are not found in patients with hypoparathyroidism after neck surgery [2]. Antiparathyroid antibodies have been reported in a pediatric population with idiopathic hypoparathyroidism [5], and in a subset of adults with the same disorder [2]. These findings suggested an autoimmune etiology, a concept further supported by the direct demonstration in vitro of a pathogenic effect in parathyroid tissue for antibodies of the IgG and IgM isotypes [2,3].

Autoimmunity is characterized by activation of $T$ cells, clonal expansion of antigen-specific $T$ cells, and production of autoantibodies by B cells [6]. If an autoimmune mechanism were operating in the pathogenesis of adult-onset idiopathic hypoparathyroidism (AOIH), it might be possible to demonstrate lymphocyte phenotype alterations consistent with T-cell activation and parathyroid antigen-specific clonal expansion. To this effect, the present study used monoclonal antibodies and flow cytometry to determine the expression of 14 different lymphocyte phenotype markers in peripheral blood cells of patients with AOIH (diagnosed after the age of 30 years), patients with Graves' disease, and normal healthy control subjects. We also investigated the mitogenic response of peripheral blood lymphocytes to parathyroid and thyroid cell surface antigens. The results, consistent with generalized immunoactivation in patients with $\mathrm{AOIH}$, provide additional information on immune alterations associated with this condition.

\section{PATIENTS AND METHODS}

The clinical subjects were collected from the Endocrine Services at Southern Illinois University, Springfield, Illinois, and at Baylor College of Medicine, Houston, Texas. There were eight patients with AOIH, 13 with Graves' disease, and 110 normal controls. The diagnosis of AOIH had been made in 
all cases by the combinations of: presentation after 30 years of age, hypocalcemia with undetectable or normal parathyroid hormone levels, and absence of previous neck surgery or systemic disease known to affect the parathyroid glands. The age of onset ranged from 36 to 71 years, while at the time of the study, patients were 36 to 78 years old. Seven of the patients were male and one was female; two had coexisting Hashimoto's thyroiditis, and one had Hashimoto's thyroiditis, Addison's disease, and hypogonadotropic hypogonadism. Six of the eight patients had antibodies against parathyroid tissue, as detected by direct immunofluorescence [2]. At the time of the study, most patients were receiving vitamin D and calcium supplementation and had normal blood calcium and phosphate levels.

To match the presumed heterogeneity of duration of idiopathic hypoparathyroidism, the Graves' disease group included five patients with active disease and eight with inactive disease, none of whom received antithyroid drugs. Patients with active Graves' disease had clinical thyrotoxicosis, and their blood samples were drawn immediately before administration of radioiodine therapy. Patients with inactive Graves' disease had been treated with ${ }^{131}$ I a year or more before testing and had developed permanent hypothyroidism. They were receiving replacement therapy with thyroid hormone. Controls were healthy individuals without known hematologic disorders; 71 were female ( 11 to 80 years old) and 39 were male (24 to 67 years old).

Blood samples were obtained locally in Springfield, Illinois, from five hypoparathyroid patients, eight of the 13 patients with Graves' disease, and 60 controls. Samples from the remaining patients and controls were shipped from source in insulated styrofoam containers by overnight air express. All blood specimens were collected in EDTA-anticoagulated Vacutainers (Becton Dickinson, Rutherford, NJ) and were tested within 16 hours of venipuncture. The absolute lymphocyte count was obtained and a buffy coat was prepared by centrifugation (500×g, 10 minutes), washing and resuspending in $0.15 \mathrm{M}$ phosphate-buffered saline (containing $0.1 \%$ sodium azide [PBSA]) at a concentration of approximately 2,000 lymphocytes $/ \mathrm{mL}$.

Staining with monoclonal antibodies was performed in $12 \times 75-\mathrm{mm}$ disposable glass tubes by adding $0.1 \mathrm{~mL}$ of washed, buffy coat lymphocytes to the manufacturer-suggested working volume of monoclonal antibodies, and incubating in the dark at $4^{\circ} \mathrm{C}$ for 30 minutes [7]. Unbound monoclonal antibodies were removed by two washes in PBSA (500 $\mathrm{Kg}, 3$ minutes), and erythrocytes were lysed according to the Becton Dickinson protocol (Becton Dickinson, Mountain View, CA; source book, sec- tion 2.11). Lymphocytes were washed again in PBSA and fixed in 1\% paraformaldehyde in PBSA for flow cytometry analysis. This was performed with a flow cytometer using a Biohazard flow tube and a $5-W$ laser exciting at $488 \mathrm{~nm}$ (Coulter Epics C, Coulter Immunology, Hialeah, FL).

Monoclonal antibodies were labeled with fluorescein isothiocyanate (FITC) or phycoerythrin (PE), and the test panel included: CD45 (FITC)/CD14 (PE) (Coulter) for use in gating of lymphocytes (CD45) and exclusion of monocytes (CD14); T4 (FITC) (Coulter) and Leu-3 (FITC) (Becton Dickinson) to detect CD4 helper T cells; Leu-2 (PE) (Becton Dickinson) to detect CD8 suppressor/cytotoxic lymphocytes; IL2R (FITC) (Becton Dickinson) to detect interleukin-2 receptor-bearing $T$ lymphocytes (used with CD5 [T1 PE] [Coulter] to restrict analysis to T cells); TA1 (FITC) (Coulter) to detect activated $\mathrm{T}$ cells (CD26) in conjunction with $\mathrm{CD} 5$ as above; $2 \mathrm{H} 4$ (PE) (Coulter) to detect the suppressor/inducer phenotype (CD45RA) on helper T cells (CD4); 4B4 (PE) (Coulter) to detect the inducer of helper phenotype CD29 on CD4 cells; Leu-11b (FITC) (Becton Dickinson) to detect the $\mathrm{Fc}$ receptor on natural killer and $\mathrm{K}$ cells (CD16); and NKH-1 (PE) (Coulter) to detect natural killer cells (CD56); Leu-4 (FITC)/DR (PE) (Becton Dickinson) and I3 (FITC)/T3 (PE) (Coulter) to detect activated T cells coexpressing the MHC class II DR antigen. Isotype controls included: mouse IgG1 (both FITC- and PE-labeled) and IgG2a (FITC and $\mathrm{PE})$.

After the bit map and color compensation were set, a minimum of 2,000 lymphocytes were analyzed for each phenotype determination.

\section{Tissue Collection}

Samples of adenomatous parathyroid glands, Graves' disease (not treated with antithyroid drugs) thyroid glands, and normal liver were obtained at the time of surgery, snap-frozen in liquid nitrogen, and stored at $-70^{\circ} \mathrm{C}$ until tested. Plasma membranes produced by mechanical homogenization were separated from nuclei by centrifugation (1,500×g, 20 minutes). The supernatant was then centrifuged on a continuous Percoll gradient $\left(30,000 \times g, 25\right.$ minutes) and fractions containing $5^{\prime}$ nucleotidase were pooled and used. Protein concentration was measured by a modification of the Coomasie brilliant blue dye-binding assay [8]. Purified bovine thyroglobulin was obtained from a commercial source (Sigma, St. Louis, MO).

\section{Lymphocyte Stimulation Assay}

Peripheral blood mononuclear cells (PBMC) were obtained by standard Ficoll-Hypaque centrifugation methods from two patients with AOIH: one 


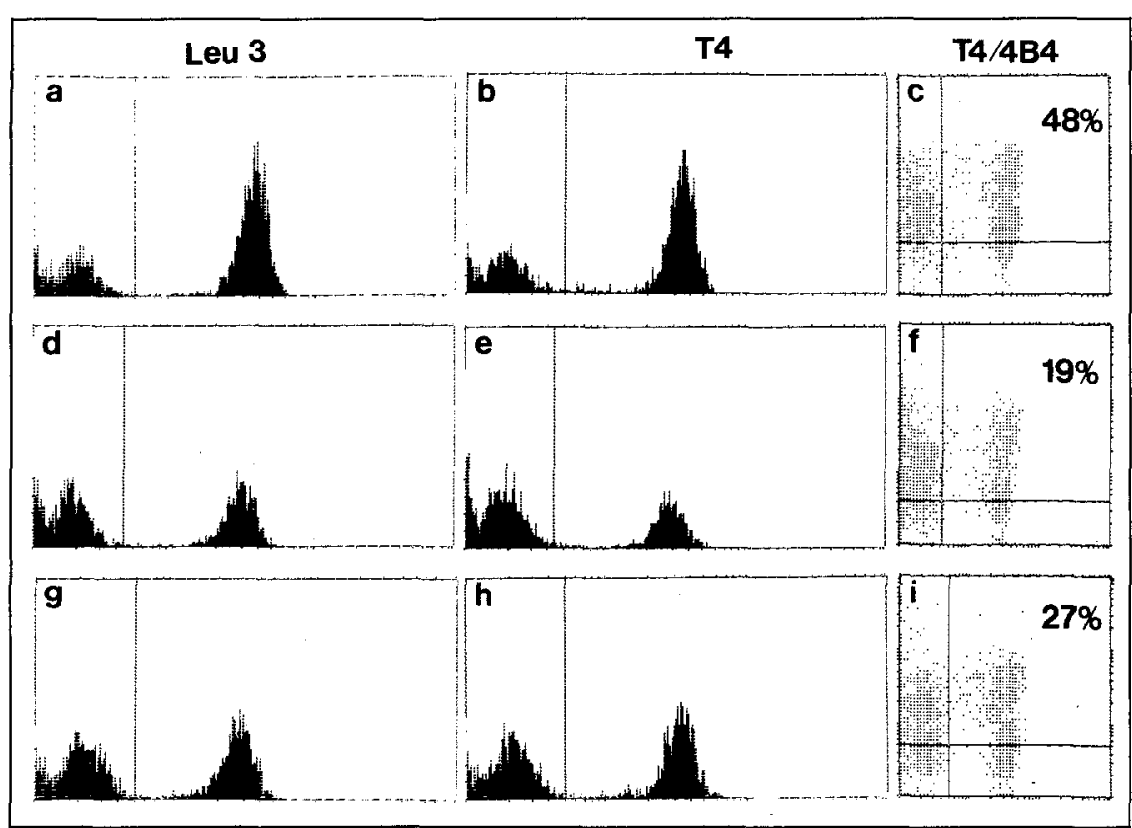

Figure 1. Representative histograms of flow cytometry demonstrating intraindividual consistency for CD4 (as detected by Leu-3 and T4 antibodies) and CD4/CD29 (T4/4B4). Histograms a, b, and c correspond to a patient with $\mathrm{AOIH}$ d, e, and $f$ to a patient with Graves' disease; and $\mathbf{g}, \mathbf{h}$, and $\mathbf{i}$ to a normal control subject. On histograms $\mathbf{c}$, $\mathbf{f}$, and $\mathbf{i}$, the percent value in the upper right quadrant represents double-stained lymphocytes; the horizontal axes represent relative fluorescence intensity and the vertical axes the number of cells positive. The horizontal axes of the quadrant histograms represent green fluorescence intensity and the vertical axes red fluorescence intensity. with isolated hypoparathyroidism (Patient 1 ) and the other with hypoparathyroidism and hypothyroid Hashimoto's thyroiditis (Patient 2). The PBMC were cultured in RPMI-1640 tissue culture medium (Hepes buffer) as described previously [7]. The PBMC were cultured with the following mitogens: phytohemagglutinin (PHA) $0.5 \mu \mathrm{g} / \mathrm{mL}$, parathyroid cell membranes at protein concentrations of 1,10 , and $20 \mu \mathrm{g} / \mathrm{mL}$, thyroid cell membranes at 20 $\mu \mathrm{g} / \mathrm{mL}$, thyroglobulin at $10 \mu \mathrm{g} / \mathrm{mL}$, liver cell membranes at $20 \mu \mathrm{g} / \mathrm{mL}$, and phosphate-buffered saline as a background control. After 72 hours of incubation in $5 \%$ carbon dioxide in air at $95 \%$ humidity at $37^{\circ} \mathrm{C}$, the PBMC cultures were pulsed for 18 hours with one $\mu \mathrm{Ci} /$ well of ${ }^{3} \mathrm{H}$-thymidine (New England Nuclear, Boston, MA), harvested onto glass fiber filter papers, and washed. The ${ }^{3} \mathrm{H}$ incorporation was determined by scintillation spectroscopy. All cultures were performed in quadruplicate.

\section{Statistical Analysis}

The statistical significance of differences between groups was evaluated by one-way analysis of variance (ANOVA). The Tukey-HSD procedure was used as a follow-up test; the significance level was set at $p=0.05$.

\section{RESULTS}

Typical flow cytometry patterns for CD4 and CD29 in representative patients for each clinical group are presented in Figure 1. The horizontal axes of the single-color histograms, i.e., $a$ and $b$, represent fluorescence intensity increasing to the right, and the vertical axes represent the proportion of measured events at each fluorescence intensity. Events to the right of the vertical cursors are con- sidered positive. In the two-color histograms, i.e., c, the horizontal axes represent relative intensity of green fluorescence and the vertical axes red fluorescence. The vertical and horizontal cursors discriminate between background and positive events. Events in the upper right quadrants represent cells with both green and red fluorescence.

Of the 13 lymphocyte phenotype frequencies tested in the three groups, there were no significant differences between any pairs of the three groups for the following: CD8, CD3, CD26/CD4 (coexpressed), CD45RA/CD4, CD16/CD56, and the ratio of CD4:CD8. Of the two monoclonal antibody combinations used to detect CD3/DR, I3/T3 (Coulter) and Leu-4/DR (Becton Dickinson), the frequency of Leu-4/DR did not differ significantly in any comparison (Table I), but the I3/T3 combination was significantly affected by diagnostic category.

Significant differences ( $p<0.05$, one-way ANOVA) between controls and patients with $\mathrm{AOIH}$ were observed for the following phenotypes (Table II): CD4 (helper T cells); CD29/CD4 (inducer of helper T cells); and CD16 and CD56 (natural killer and K cells). The frequencies of all these phenotypes were significantly higher in patients with AOIH than in control subjects. An activation marker for T cells, the expression of DR, was also significantly elevated in the AOIH group, but only when assessed with the Coulter monoclonal antibodies I3/T3.

The CD5/CD20 phenotype, a presumed marker for B cells involved in autoimmunity [9], was also tested in two of the hypoparathyroid patients and found within the control range (data not shown).

No lymphocyte phenotype frequency differed significantly between the patients with active and inactive Graves' disease, but when both groups of 


\begin{tabular}{|c|c|c|c|}
\hline \multicolumn{4}{|c|}{$\begin{array}{l}\text { TABLE I } \\
\text { Lymphocyte Phenotype Frequencies Not Differing Significantly } \\
\text { Between Groups* }\end{array}$} \\
\hline \multirow[b]{2}{*}{ Phenotype } & \multicolumn{3}{|c|}{$\%$ Total Lymphocytes Positive } \\
\hline & Hypoparathyroid & Graves' & Controls \\
\hline CD3 & $71.8(8.6)$ & $60.6(17.3)$ & $62.6(12.6)$ \\
\hline $\mathrm{CD} 8$ & $2 /(10.5)$ & $20.7(7.1)$ & $23.1(7.1)$ \\
\hline $\mathrm{CD} 4: \mathrm{CD} 8$ & $2.25(1.12)$ & $2.22(0.81)$ & $1.87(0.73)$ \\
\hline & $11.9(4.5)$ & $9.4(5.2)$ & $11.4(4.9)$ \\
\hline CD3/DR (Leu-4) & $2.5(2.1)$ & $2.1(2.2)$ & $1.9(1.1)$ \\
\hline$/$ CD5 & $9.9(3.8)$ & $13.5(5.3)$ & $10.4(3.8)$ \\
\hline CD56 & $12.7(13.6)$ & $8.2(4.7)$ & $8.8(3.4)$ \\
\hline AA/CD4 & $27.9(12.9)$ & $19.8(11.8)$ & $21.8(11.5)$ \\
\hline
\end{tabular}

${ }^{*} \mathrm{p}>0.05$ for all intergroup differences (one-way anaiysis of variance) tStandard deviation in parentheses.

Graves' patients were considered together, several phenotype frequencies differed significantly from those in the normal controls. Thus, Graves' disease was characterized by T-cell activation as demonstrated by significantly higher than control frequencies of $\mathrm{CD} 25$ (the interleukin-2 receptor marker), CD26 (the activated T-cell marker), and CD3/DR (I3/T3). Only one phenotype, CD29/CD4 (inducer of helper), differed significantly between the patients with Graves' disease and the hypoparathyroid patients (Table II).

The mitogenic responses of lymphocytes to tissue membrane fractions determined in two patients with AOIH are presented in Figures 2 and 3. In Patient 1, parathyroid membranes did not stimulate ${ }^{3} \mathrm{H}$-thymidine incorporation nor did any of the other membrane fractions tested (Figure 2). Patient

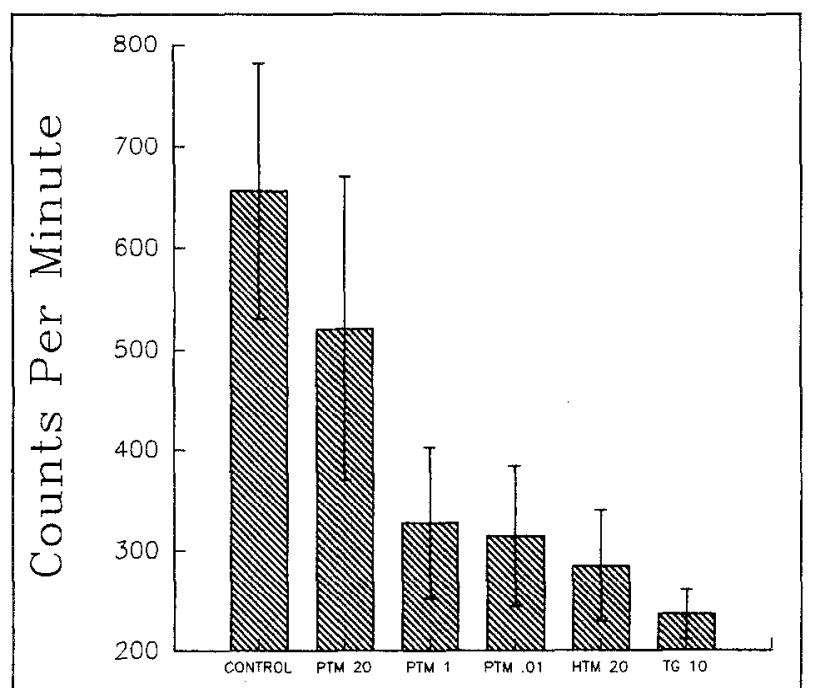

Figure 2. Lymphocyte proliferation response $\left({ }^{3} \mathrm{H}\right.$-thymidine incorporation) to parathyroid and thyroid membrane and thyroglobulin (TG) in a patient with isolated $\mathrm{AOIH}$. Antigen concentration is expressed in $\mu \mathrm{g}$ protein $/ \mathrm{mL}$. None of the responses differed significantly from those in controls $(p$ $>0.05)$. PTM = parathyroid membrane; HTM = human thyroid membrane.

\begin{tabular}{|c|c|c|c|}
\hline \multicolumn{4}{|c|}{$\begin{array}{l}\text { Lymphocyte Phenotype Frequencies Differing Significantly Between } \\
\text { Groups }\end{array}$} \\
\hline \multirow[b]{2}{*}{ Phenotype } & \multicolumn{3}{|c|}{ \% Total Lymphocytes Positive ${ }^{\star}$} \\
\hline & Hypoparathyroid & Graves' & Controls \\
\hline CD4 & $52.3(6.3)^{\dagger}$ & $42.4(10.9)$ & $40.1(9.8)^{\dagger}$ \\
\hline CD29/CD4 & $40.4(6.3)^{\dagger \grave{t}}$ & $30.4(11.4)^{\dagger}$ & $32.1(8.2)^{\ddagger}$ \\
\hline CD25 & $6.7(3.1)$ & $8.2(3.7)^{\dagger}$ & $4.8(3.9)^{\dagger}$ \\
\hline CD26 & $14.3(5.8)$ & $16.4(5.2)^{\dagger}$ & $10.8(6.6)^{\dagger}$ \\
\hline CD3/DR & $9.1(4.7)^{\ddagger}$ & $6.9(4.1)^{\dagger}$ & $3.2(1.8)^{\dagger \neq}$ \\
\hline $\mathrm{CD} 16$ & $16.0(17.7)^{\dagger}$ & $13.6(8.2)$ & $8.7(6.0)^{\dagger}$ \\
\hline CD56 & $18.3(16.1)^{\dagger}$ & $11.2(5.0)$ & $9.7(6.1)^{\dagger}$ \\
\hline
\end{tabular}

*Standard deviation in parentheses.

t+Denotes pairs of groups that differ significantly at $p<0.05$, by one-way analysis of variance.

2, who had Hashimoto's thyroiditis, demonstrated a slight but not statistically significant mitogenic response to thyroglobulin and absent response to thyroid or parathyroid membranes (Figure 3). Both subjects responded significantly to PHA $0.5 \mu \mathrm{g} / \mathrm{mL}$ (500-fold increase) but not to liver membranes (data not shown).

\section{COMMENTS}

The present study, performed on peripheral lymphocytes from patients with $\mathrm{AOIH}$, shows a pattern of T-cell activation. Thus, the accumulated evidence suggests a syndrome of immune dysfunction in $\mathrm{AOIH}$, in common with the recognized autoimmune disorder Graves' disease [10]. Most importantly, patients with AOIH have a higher than normal frequency of the CD3/DR phenotype representing T-cell activation. Although the natural killer phenotype frequency was also increased in

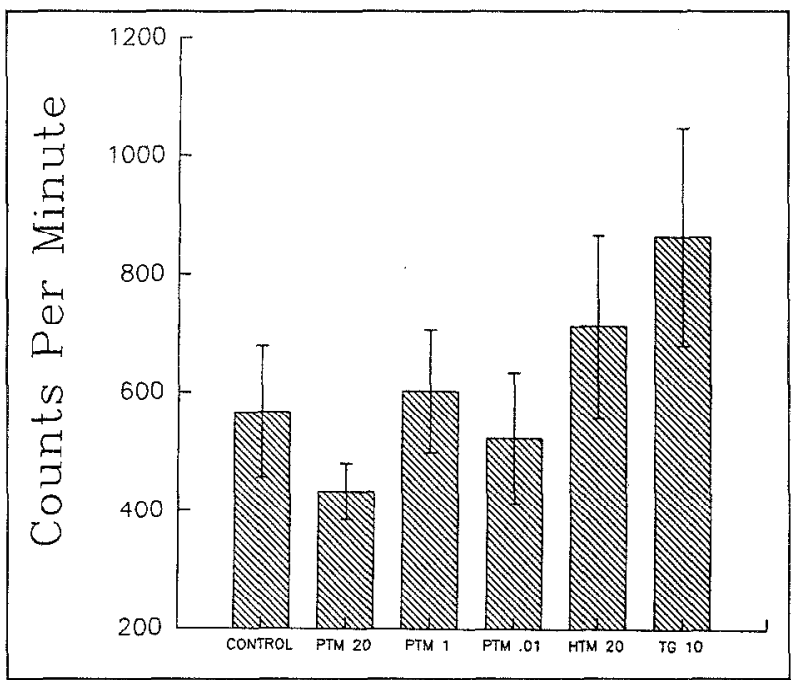

Figure 3. Lymphocyte proliferation response in a patient with $\mathrm{AOIH}$ and Hashimoto's thyroiditis. None of the responses were significantly different from those in controls. PTM = parathyroid membrane; HTM = human thyroid membrane; $T G=$ thyroglobulin. 
AOIH, the significance of this finding is unclear. Natural killer cells [11] have recently been found to bind thyrotropin (TSH) in vitro [12], but specific TSH receptors were not characterized in that study [12].

Evidence for B-cell activation was not observed in this study, despite the high incidence of autoantibodies known to be present in patients with idiopathic hypoparathyroidism in general [4], and especially in AOIH [2]. However, caution must be taken in interpreting this finding, since B-cell activation was also absent in Graves' disease, where immunoglobulins against the TSH receptor are the direct cause of hyperthyroidism [10]. Moreover, the presence of polyreactive IgM autoantibodies may not necessarily imply autoimmune disease, since such antibodies have been found to occur naturally in nonimmunized mice and in humans $[13,14]$.

An important question that remains unanswered is the nature of the antigenic stimulus that triggers T-cell activation in AOIH. If that were primarily determined by a parathyroid component, it would be difficult to understand the observed generalized immune activation in response to such a small antigen mass. We nevertheless performed additional experiments specifically addressed to evaluating such a possibility. Using viable lymphocytes, as confirmed by their appropriate response to PHA, we did not find mitogenic responses in $\mathrm{AOIH}$ lymphocytes co-cultured with parathyroid cell membranes. Most likely, these findings imply that peripheral lymphocytes are not actively involved in the pathogenesis of the hypoparathyroidism but reflect an underlying state of nonspecific immune activation. Alternate possibilities include the absence of a normal constituent (parathyroid membrane antigen) from the parathyroid adenoma-derived preparation used to stimulate the lymphocytes, or immune activation by a parathyroid antigen not accessible to the lymphocytes without prior enzymatic digestion or other procedures that may unmask a cryptic antigen.

Surprisingly, the generalized T-cell activation was more extensive in idiopathic hypoparathyroidism than in Graves' disease. Although the lymphocyte phenotype pattern generally reflects activation in Graves' disease $[10,15,16]$, subset frequencies in that disease were found to be intermediate between AOIH and controls. Of note, no differences in T-cell phenotypic profiles were noted between active and quiescent Graves' disease, an area in which consensus has not been reached [10].

Taken together, the present findings and previous reports [1-4] further help define AOIH. While the idiopathic label still remains for the primary trigger of the disease, it now appears that the immune system may be consistently abnormal, per- haps as the main mediator or at least a significant contributor in the pathogenesis of hypoparathyroidism. Clearly, T-cell activation and a humoral reaction are also components of $\mathrm{AOIH}$, with the importance of the latter having already received partial confirmation. Thus, circulating antiparathyroid antibodies do have pathogenic activity in vitro, as documented by the production of parathyroid cell lysis [3] or interference with the signal recognition/transduction mechanism responsible for calcium-regulated parathyroid hormone secretion [2].

In conclusion, the novel feature of T-cell activation in AOIH should be added to the immune abnormalities already reported in these patients. The present results suggest that AOIH may be the result of a generalized immune disturbance in which hypoparathyroidism is one manifestation.

\section{REFERENCES}

1. Wortsman J, Posillico JT, Brown EM, Eisenbarth GS, Mallette LE. Case 34-198 [letter]. N Engl J Med 1988; 318: 857.

2. Posillico JT, Wortsman J, Srikanta S, Eisenbarth GS, Mallette LE, Brown EM. Parathyroid cell surface autoantibodies that inhibit parathyroid hormone secretion from dispersed human parathyroid cells. J Bone Miner Res 1986; 1: 475-83.

3. Brandi ML, Aurbach GD, Fattorossi A, Quarto R, Marx SJ, Fitzpatrick LA. Antibodies cytotoxic to bovine parathyroid cells in autoimmune hypoparathyroidism. Proc Natl Acad Sci USA 1986; 83: 8366-9.

4. Betterle C, Caretto A, Zeviana M, Pedini B, Salviata C. Demonstration and characterization of anti-human mitochondria autoantibodies in idiopathic hypoparathyroidism and in other conditions. Clin Exp Immunol 1985; 62: 353-60. 5. Blizzard RM, Chee D, Davis $W$. The incidence of parathyroid and other antibodies in the sera of patients with idiopathic hypoparathyroidism. Clin Exp Immunol 1966; 1: 119-28.

6. Wilkin TJ. Receptor autoimmunity in endocrine disorders. N Engl J Med 1990 323: $1318-24$

7. Wortsman J, McConnachie P. Baker JR Jr, Burman KD. Immunoglobulins that cause thymocyte proliferation from a patient with Graves' disease and an enlarged thymus. Am J Med 1988; 85: 117-21.

8. Wen L, Peakman M, Lobo-Yeo A, et al. T-cell-directed hepatocyte damage in autoimmune chronic active hepatitis. Lancet 1990; 336: 1527-30.

9. Hayakawa K, Hardy RR, Honda M, Herzenberg LA, Steinberg AD, Herzenberg LA. Ly-1B cells: functionally distinct lymphocytes that secrete IgM autoantibodies. Proc Natl Acad Sci USA 1984; 81: 2494-8.

10. Burman KD, Baker JR Jr. Immune mechanisms in Graves' disease. Endocr Rev 1985; 6: 183-232.

11. Abruzzo LV, Rowley DA. Homeostasis of the antibody response: immunoregulation by NK cells. Science 1983; 222: 581-7

12. Coutelier J-P, Kehrl JH, Bellur SS, Kohn LD, Notkins AL, Prabhakar BS. Binding and functional effects of thyroid stimulating hormone on human immune celis. J Clin Immunol 1990; 10: 204-10.

13. Hartman AB, Mallett CP, Srinivasappa J, Prabhakar BS, Notkins AL, Smith Gill SJ. Organ reactive autoantibodies from non-immunized adult BALB/C mice are polyreactive and express non-biased VH gene usage. Mol Immunol 1989; 26: 359-70.

14. Allaway GP, Srinivasappa J, Miller FW, Prabhakar BS, Notkins AL. Spontaneously proliferating human B lymphocytes make autoantibodies. J Infect Dis 1988; 157: 968-72.

15. Bagnasco M, Venuti D, Paolieri F, Torre G, Ferrini S, Canonica GH. Phenotypic and functional analysis at the clonal level of infiltrating $T$ lymphocytes in papillary carcinoma of the thyroid: prevalence of cytolytic $T$ cells with natural killer-like or lymphokine-activated killer activity. J Clin Endocrinol Metab 1989; 69: 832-6.

16. Guerin V, Bene MC, Amiel C, Hartemann P, Leclere J, Faure G. Decreased lymphocyte function-associated antigen-1 molecule expression on periphera blood lymphocytes from patients with Graves' disease. J Clin Endocrinol Metab 1989; 69: 648-53. 